\title{
Bus Attendance System using Optical Character Recognition
}

\author{
P. Bhavani, S. Vaishnavi, P. Vennila, V. Vijayalakshmi
}

\begin{abstract}
In today's world managing the records of attendance of staffs, students, employee or bus is a tedious task. This project focuses on automating the bus attendance process through vehicle license plate recognition. As, the license plate is a feature that is peculiar to every vehicle, it would help in efficiently marking the bus attendance. The bus attendance system using RFID is a time consuming process. Hence we developed a project to efficiently mark attendance using number plate recognition and OCR. The system was trained using faster RCNN model with bus image dataset. The proposed system is the number plate is captured through surveillance camera and the captured image will be passed as an input to the neural network for training and the number plate will be detected. Character extraction is done using OCR and extracted character matched will be checked with the database and the attendance for particular bus will be marked.

Keywords : Bus attendance system, faster RCNN, Number plate detection, $\mathrm{OCR}$.
\end{abstract}

\section{INTRODUCTION}

Number plate recognition is a high accurate system which is capable of reading the license plate without any human intervention through the use of image capturing, detection of characters from the image given character recognition to convert image to text and decoded text of that plate. Using picture as input, features of number plate can be predicted. No wonder, the computer also trains in such a way The relationship between data will be identified with the help of neural networks. It works similar to the human brain. The architecture of neural network is same as the arrangement of neurons present in the brain.With the number of vehicles being increased, it is identified that the systems for identifying the vehicle is also increasing parallel. Highway speed detection, safety, light detection, stolen vehicle detection,

Revised Manuscript Received on April 25, 2020.

* Correspondence Author

P.Bhavani*, Assistant Professor, Department of Computer Science and Engineering, Sri Manakula Vinayagar Engineering College, Puducherry, India. Email: bhavani.smvec@gmail.com

S.Vaishnavi, Department of Computer Science and Engineering, Sri Manakula Vinayagar Engineering College, Puducherry, India. Email: vaishusv1999@gmail.com

P.Vennila, Department of Computer Science and Engineering, Sri Manakula Vinayagar Engineering College, Puducherry, India. Email: pvennila72@gmail.com

V.Vijayalakshmi, Department of Computer Science and Engineering, Sri Manakula Vinayagar Engineering College, Puducherry, India,Email:sekarviji1998@gmail.com

(C) The Authors. Published by Blue Eyes Intelligence Engineering and Sciences Publication (BEIESP). This is an open access article under the CC BY-NC-ND license (http://creativecommons.org/licenses/by-nc-nd/4.0/) automatic switching systems, and human and non-human loss collection systems are some of the applications of vehicle identification system. Using the automatic license plate recognizing system the manual power is reduced since the process is automated. In order to obtain the accurate number plate recognition system it mainly focuses on three topics. They are, finding the location of the panel of input images, character segmentation from the input image and the visual character recognition [2]. The first and foremost step is to determine the position of the number plate in input picture. Using structural analysis and color analysis method the localization of plate can be recognised. Using connected component analysis unwanted spots can be removed. This system is cost effective and fast. Number plate recognition is a compiled system which identifies the number plate from the captured image. There are many applications using this system and is mainly used in traffic controls and in toll gates. These systems automate the process of identifying vehicle license number and make the process easier.

\section{RELATED WORKS}

Chirag Patel [1] different methods of recognizing a number plate by taking into account image size, success rate and processing time as parameters. At the end they have suggested an extension to plate recognition. For the purpose of detecting number plate the following aspects should be examined.

The first one is the plate can be of different size and can be positioned in any place in the vehicle. While moving on to background and screw it can have different background color and a screw may be considered as a character.

However character segmentation was not accurate. So B. Suresh [16] proposed character separation by template matching. For feature extraction they proposed to find, mark and save all features from the segmented number plate. Template is nothing but a region of a sub-photograph inside a picture.

Template matching works by figuring out the resemblance between given template and home window of the same image. The comparison between two methods of character recognition is shown.

Image processing helps in processing digital imaging by means of a computer. R. Prithvi [22] proposed automation of the number plate recognition to achieve accuracy greater than 95\%. From the imaging devices and sensors connected to Raspberry Pi model we can acquire data.

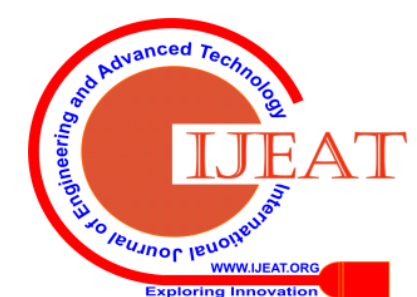




\section{Bus Attendance System using Optical Character Recognition}

The training of neural network can be done using feed-forward, back propagation algorithm. The network has 1650 input unit, 36 output unit and 500 hidden units. After optimizing the neural network with respect to regularization parameter, number of training steps and number of hidden layer neurons, 500 layers was chosen. 26 English alphabets (A-Z) and ten numbers (0-9) have been trained using this network. Depending upon the environment at which the system is implemented the region of interest has been predefined.

They designed this system for cars and can be extended to other vehicles. Finally they concluded that the algorithm poorly performed under brightly illuminated environments.

DVR. Mohan [2] proposed searching for license plate recognition using three major steps: number pad space, character segmentation, character recognition. Efficiency can be improved in each step. Adaptive threshold can be used to highlight a characters and the background can be suppressed.

Image scissoring algorithm can be used to segment the OCR engine which is known as tessaract that returns ASCII to the license number. Another method they suggested is to deploy feed- forward background for character classification and backward propagation algorithm can be used for developing the neural network. The problem of character fragmentation can be addressed by horizontal and vertical graph and component survey. In this proposal the picture is captured in RGB format and converted to a gray image.

After the conversion of the image preprocessing is done by threshold algorithm. The gray scale image is converted into binary image by using the distance formula. The plate can extracted by using either a shape analysis or color analysis. In shape analysis, the algorithm looks for a rectangular proportion whereas in the color analysis the algorithm looks for white or yellow number plates.

The corners are marked and connected with box and all areas of interest are extracted. Next the segmentation of character is done and it is recognized. Finally they concluded the future extension of this work can be developed by using template matching algorithm.

M V Raghunadh proposed the bounding box method for the number plate recognition and detection. The input image is selected and the noise is removed in the particular area of image. Then the edge of the plate is detected by using edge detection method and then the segmentation for each character is done individually. The four main steps followed here is

$$
\begin{aligned}
& \text { - Preprocessing } \\
& \text { - License plate localization } \\
& \text { - Character recognition } \\
& \text { - Character segmentation }
\end{aligned}
$$

\section{PROPOSED SYSTEM}

The overview of the system is connecting a web page to a computer that contains the trained model. The model will be trained using faster recurrent neural network that can extract the features of the bus license plate and convert the text present in the plate.

A surveillance camera is connected which captures image and sends it to the computer. The number plate is detected from the bus and compared with the previously detected number plate. The detected number plate will be passed to the OCR algorithm. The OCR algorithm extracts the text from the detected number plate. The output of the OCR is compared with the previously entered bus details in the database. If the license plate number matches it marks the attendance for the particular bus in the excel sheet.

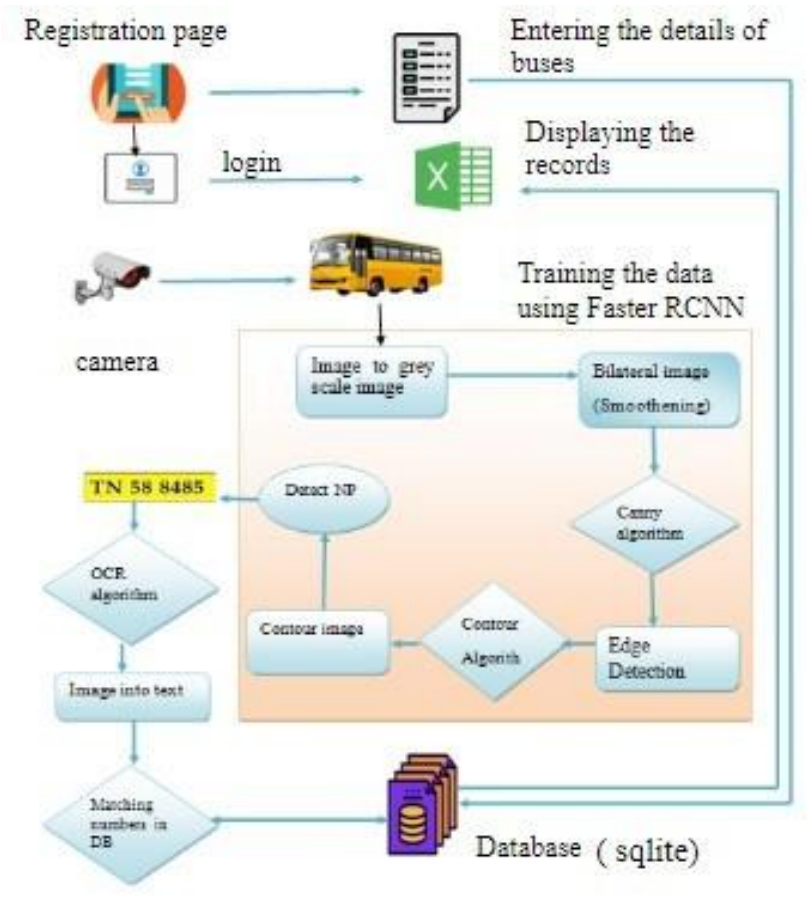

Fig1. Proposed system architecture

It can be programmed using python3 the camera and the trained model is connected and by using computer vision library the information related to the input image can be gathered.

The camera captures the image of the bus while entering the entrance. The frames of the images are individually analyzed for detecting number plate.

The faster RCNN is used for training the dataset. The entered details will be stored in the database and a DB file will be generated. From this file the excel sheet can be generated.

The modules of this proposed work are:

- Data Preprocessing

- Model Training

- Database Connectivity

- Testing Phase

\section{A. Data Preprocessing}

The dataset consisting of bus images is chosen for this work. The dataset has more than 20000 images covering almost all the license plate of a vehicle. These data are preprocessed before it is trained. Through preprocessing the noise detection can be achieved.

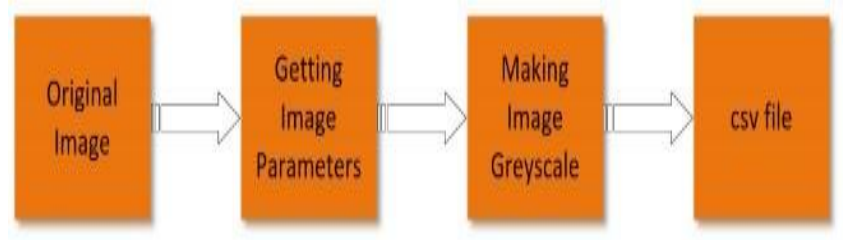

Fig2. Dataset Processing

Published By:

Blue Eyes Intelligence Engineering \& Sciences Publication

(c) Copyright: All rights reserved. 


\section{B. Model Training}

Once the dataset is preprocessed the faster RCNN is used for training. The total data set is divided into two $80 \%$ of data set is for training and remaining $20 \%$ for testing. The dataset image is downloaded as CSV file and then the TF record for each CSV file is generated. The model is trained with the help of CNN background. Finally we obtained a trained model which can recognize the number plate.

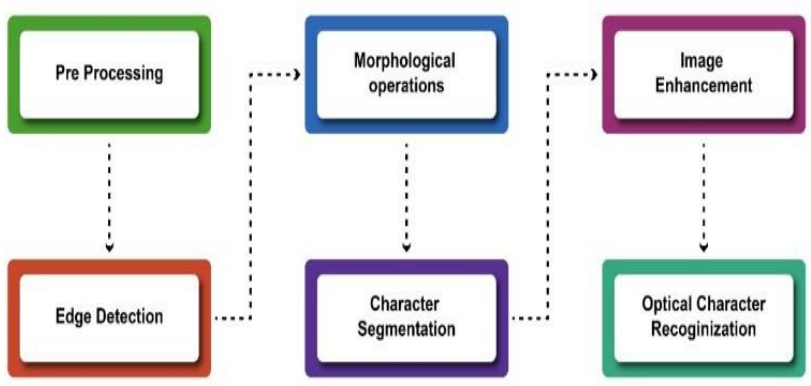

Fig3. Model Training

\section{Database Connectivity}

The trained model is connected to the database which contains all the bus details of the particular organization. The character extracted from the OCR will be compared with the bus details stored in the database. If the both record matches then the bus attendance will be marked in the excel sheet. The working of OCR is shown in the figure 4.
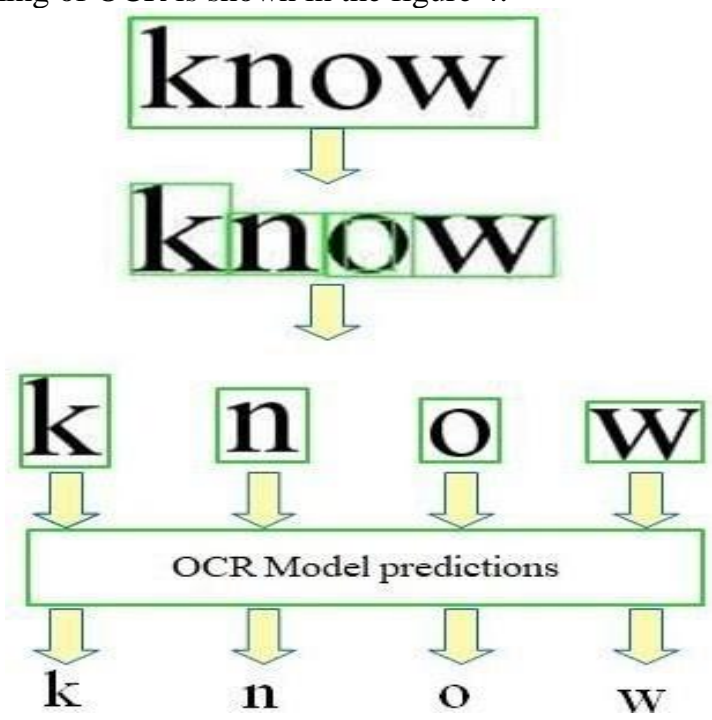

Fig4. Character Segmentation

\section{Testing Phase}

The surveillance camera is used for testing in real time. The images are captured and processed against the trained model. The model will compare the input images that exactly match with already trained data. Finally the attendance will be marked and stored in the excel sheet.

\section{EXPERIMENTAL RESULTS}

The system uses Faster RCNN which is more accurate than the Convolutional Neural Network. Moreover the attendance is marked perfectly.

The system has detected the bus number plate and the attendance has been marked. Fig 5 and Fig 6 shows the pictorial representation of the marked attendance.

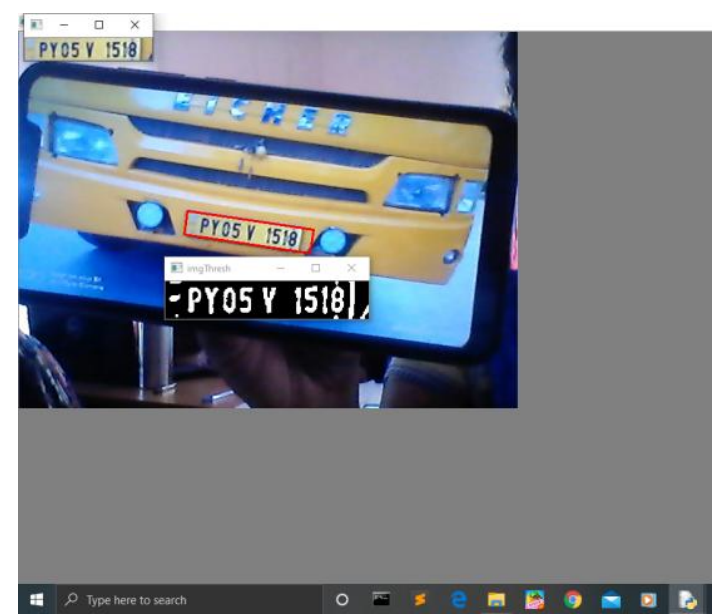

Fig 5. Experimental results for number plate detection

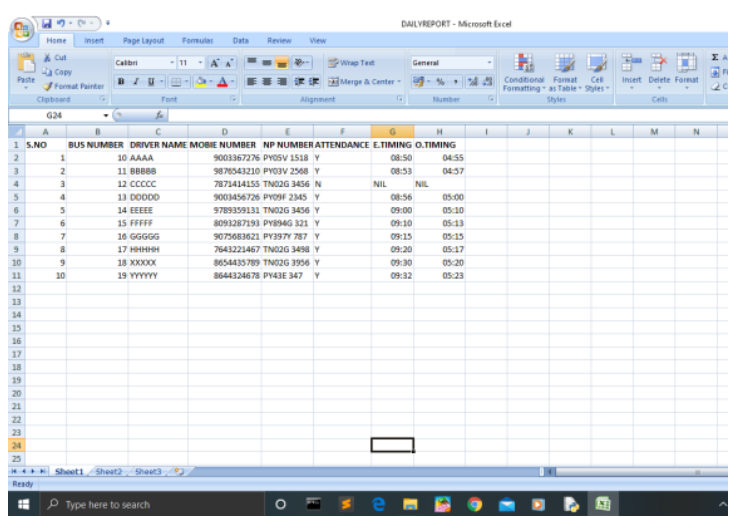

Fig 6. Experimental results for marking attendance

\section{CONCLUSION}

In this project an efficient hierarchical Bus Attendance System. In proposed system we connected to the database in order to maintain the bus attendance. The recognition is done and with the matched details the bus attendance is marked. The accuracy can be advanced significantly by using Faster RCNN algorithm. The CNN algorithm has many drawbacks, so we are using Faster RCNN algorithm to detect the number plate. By using this algorithm the accuracy can be increased.

\section{REFERENCES}

1 Dipti Shah, Chirag Patel and Atul Pate, Automatic Number Plate Recognition System, International Journal of Computer Applications (0975 - 8887) Volume 69- No.9, May 2013.

2 Tella Pavani and DVR. Mohan, Number plate recognition using OPENCV-PYTHON, Volume: 06, Issues: 03| March 2019.

3 You-Shyang Chen and Ching-Hsue Cheng, "A Delphibased rough sets fusion model for extracting payment rules of vehicle license tax in the government sector, "Expert Systems with Applications, vol. 37, no. 3, pp. 2161-2174, 2010.

4 Anton Satria Prabuwono and Ariff Idris, "A Study of Car Park Control System Using Optical Character Recognition," in International Conference on Computer and Electrical Engineering, 2008, pp. 866-870.

5 An Albiol, L Sanchis, and J.M Mossi, "Detection of Parked Vehicles Using Spatiotemporal Maps," IEEE Transactions on Intelligent Transportation Systems, vol. 12, no. 4, pp. 1277- 1291, 2011.

6 Christos Nikolaos E. Anagnostopoulos, Ioannis E. Anagnostopoulos, Ioannis D. Psoroulas, Vassili Loumos, and Eleftherios Kayafas, License Plate Recognition From Still Images and Video Sequences: A Survey, vol. 9, no. 3, pp. 377- 391, 2008. 


\section{Bus Attendance System using Optical Character Recognition}

7 H. Erdinc Kocer and K. Kursat Cevik, "Artificial neural network based vehicle license plate recognition," Procedia Computer Science, vol. 3, pp. 1033-1037, 2011.

8 A Roy and D.P Ghoshal, "Number Plate Recognition for use in different countries using an improved segmentation," in $2^{\text {nd }}$ National Conference on Emerging Trends and Applications in Computer Science(NCETACS), 2011, pp. 1-5.

9 Kaushik Deb, Ibrahim Kahn, Anik Saha, and Kang-Hyun Jo, "An Efficient Method of Vehicle License Plate Recognition Based on Sliding Concentric Windows and Artificial Neural Network," Procedia Technology, vol. 4, pp. 812-819, 2012.

10 S. Du, M. Ibrahim, M. Shehata, and W. Badawy. Automatic license plate recognition (alpr): A state-of-the-art review. Circuits and Systems for Video Technology, IEEE Trans. on, 23(2):311-325, 2013.

11 J.Redmon and A.Farhadi. Yolo9000: Better, faster, stronger arxiv preprint arXiv: 1612.08242, 2016.

12 T.-Y. Lin, M. Maire, S. Belongie, J. Hays, P. Perona, D. Ramanan, P. Dolla'r, and C. L. Zitnick. Microsoft COCO: Common objects in context. In ECCV. 2014.

13 W. Liu, D. Anguelov, D. Erhan, C. Szegedy, S. Reed, C.- Y. Fu, and A. C. Berg. Ssd: Single shot multibox detector.In European Conference on Computer Vision, pages 21- 37.Springer, 2016.

14 Y. LeCun, L. Bottou, Y. Bengio, and P. Haffner. Gradientbased learning applied to document recognition. Proc. of the IEEE, 1998.

15 Abishek Kashyap, B. Suresh, Anukul Pati and Ankit Jaiswal, Automatic Number Plate Recognition, International Conference on Advances in Computing, Communication Control and Networking(ICACCCN2018).

16 K.V.Mahesh Babu, M.V. Raghunadh, Vehicle number plate detection and recognition using bounding box method, Published in International Conference on 2016.

17 Sarbjit Kaur, An Automatic Number Plate Recognition System under Image Processing, International Journal of Intelligent System Technogies and Applications 8(3):14-25 March2016.

18 R.Radha and C.P.Sumathi, "A Novel approach to extract text from license plate of vehicle", Signal \& Image Processing: An International Journal (SIPIJ) Vol.3, No.4, August 2012.

19 Thomas K.T and Vaijayanthimala. J., A review of Automatic License plate using Edge Detection Method, International Journal for research, March-2018.

20 Hung Ngoc Do, Automatic license plate recognition using mobile device, Published in International Conference on 2016.

21 Dening Jiang, Tsehay Mekeonnen, Published in Fifth International 2012.

22 R. Prithivi, Number plate recognition system using template matching, International Journal of computer Application, January 2016.

23 Liao Yu, Research on Edge Detection in License Plate Recognition, The 2nd International Confernece on Computer Application. 5

24 T.Pratheeba, "Morphology Based Text Detection and Extraction from Complex Video Scene," International Journal of Engineering and Technology Vol.2 (3), 200 -206, 2010.

25 Saeed Rastegar, Reza Ghaderi, Gholamreza Ardeshipr \& Nima Asadi, " An intelligent control system using an efficient License Plate Location and Recognition Approach", International Journal of Image Processing (IJIP) Volume(3), Issue(5) 252, 2009.

26 Satadal Saha1, Subhadip Basu, Mita Nasipuri, Dipak Kumar Basu," License Plate Localization from Vehicle Images:An Edge Based Multistage Approach", International Journal of Recent Trends in Engineering, Vol 1, No. 1, May 2009.

27 Loumos, V.; Kayafas, E.,'License plate recognition from still images and video sequences: A survey" IEEE Transactions on Intelligent Transportation Systems, volume 9, issue 3, pages 377 -391, September 2008.

\section{AUTHORS PROFILE}

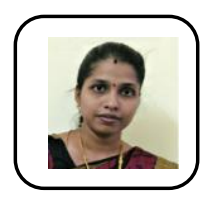

Mrs. P.Bhavani, is currently working as a Assistant Professor in the Department of Computer Science and Engineering College affiliated to Pondicherry University, Puducherry, India. She did her M.Tech in Sri Manakula Vinayagar Engineering College. She has published 4 papers in International Conference and Journal and 1 scopus.

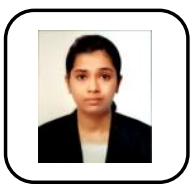

S.Vaishnavi is pursuing Undergraduate Degree in Sri Manakula Vinayagar Engineering College, Puducherry in the field of Computer Science and Engineering. She is interested in the research works on Machine Learning and Software Testing. Worked in Edge Computing where the product billing system is created for a shopping mall using machine learning algorithms. Also worked in automation testing using the Selenium tool.

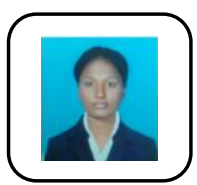

P.Vennila is pursuing Bachelor of Technology in Sri Manakula Vinayagar Engineering College, Puducherry. She is from the Computer Science and Engineering department. Her current research interests are in the field of Data science and Machine learning. Worked in a face recognition project where the age, emotion, and ethnicity of the people are identified. Also done projects on anomaly detection where the abnormal activities taking place in a pedestrian walkway are recognized.

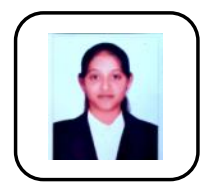

V.Vijayalakshmi is pursuing Bachelor of Technology in Sri Manakula Vinayagar Engineering College, Puducherry. She is from the Computer Science and Engineering department. Her current research interests are in the field of Data science and Machine learning. Worked in a face recognition project where the age, emotion, and ethnicity of the people are identified. Also done projects on anomaly detection where the abnormal activities taking place in a pedestrian walkway are recognized.
Published By:

Blue Eyes Intelligence Engineering

DOI: 10.35940/ijeat.D7732.049420

Journal Website: www.ijeat.org

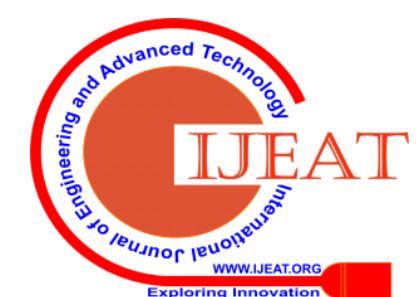

